\title{
Towards Assessing the Readability of Programming Error Messages
}

\author{
Brett A. Becker \\ University College Dublin \\ Dublin, Ireland \\ brett.becker@ucd.ie \\ Raymond Pettit \\ University of Virginia \\ Charlottesville, VA, USA \\ raymond.pettit@virginia.edu
}

\author{
Paul Denny \\ University of Auckland \\ Auckland, New Zealand \\ paul@cs.auckland.ac.nz \\ Robert Nix \\ Oklahoma Christian University \\ Edmond, OK, USA \\ robert.nix@oc.edu
}

\author{
James Prather \\ Abilene Christian University \\ Abilene, TX, USA \\ james.prather@acu.edu \\ Catherine Mooney \\ University College Dublin \\ Dublin, Ireland \\ catherine.mooney@ucd.ie
}

\begin{abstract}
Programming error messages have proven to be notoriously problematic for novices who are learning to program. Although recent efforts have focused on improving message wording, these have been criticized for attempting to improve usability without first understanding and addressing readability. To date, there has been no research dedicated to the readability of programming error messages and how this could be assessed. In this paper we examine human-based assessments of programming error message readability and make two important contributions. First, we conduct an experiment using the top twenty most-frequent error messages in three popular programming languages (Python, Java, and C), revealing that human notions of readability are highly subjective and dependent on both programming experience and language familiarity. Both novices and experts agreed more about which messages are more readable, but disagreed more about which messages are not readable. Second, we leverage the data from this experiment to uncover several key factors that seem to affect message readability: message length, message tone, and use of jargon. We discuss how these factors can help guide future efforts to design a readability metric for programming error messages.
\end{abstract}

\section{CCS CONCEPTS}

- Social and professional topics $\rightarrow$ Computing education; CS1; - Software and its engineering $\rightarrow$ Error handling and recovery; Compilers; • Human-centered computing $\rightarrow$ Human computer interaction (HCI).

\section{KEYWORDS}

compiler error messages; compiler design and implementation; CS1; enhanced error messages; HCI; novice programmers; programming; programming error messages; readability

Permission to make digital or hard copies of part or all of this work for personal or classroom use is granted without fee provided that copies are not made or distributed for profit or commercial advantage and that copies bear this notice and the full citation on the first page. Copyrights for third-party components of this work must be honored.

For all other uses, contact the owner/author(s).

ACE '21, February 2-4, 2021, Virtual, SA, Australia

(C) 2021 Copyright held by the owner/author(s).

ACM ISBN 978-1-4503-8976-1/21/02.

https://doi.org/10.1145/3441636.3442320
ACM Reference Format:

Brett A. Becker, Paul Denny, James Prather, Raymond Pettit, Robert Nix, and Catherine Mooney. 2021. Towards Assessing the Readability of Programming Error Messages. In Australasian Computing Education Conference (ACE '21), February 2-4, 2021, Virtual, SA, Australia. ACM, New York, NY, USA, 8 pages. https://doi.org/10.1145/3441636.3442320

\section{INTRODUCTION}

In a Communications of the ACM article nearly four decades ago, Brown stated that "One of the most important yet most neglected aspects of the human/machine interface is the quality of the error messages produced by the machine when the human makes a mistake" [12, p246]. A year earlier, he also wrote: "Most of us who write systems do not produce good error messages; we produce shoddy ones. Just because error messages are in some approximation to natural language, we claim that they are easy to understand. We deceive no-one but ourselves" [11, p94]. These are deceptively simple truths that are still very true today-we still misjudge and largely neglect assessing the readability of error messages [6].

It is no secret among programming educators that error messages cause substantial usability issues [17]. They have been shown to affect user behavior [25] with their effect on novices even being described as paralyzing [2]. As early as 1976, researchers were opining that programming error messages were cryptic and therefore, a barrier to learning [43]. In fact, work on improving programming error messages started as early as 1965 [37]. Nonetheless it seems that little has changed in this regard $[1,5]$.

These observations have resulted in various research efforts to "enhance" programming error messages to increase their usability (see [3, 4, 16, 19, 28, 32, 33, 41] including [6] for a comprehensive overview). However, after more than fifty years of writing about this problem, solutions are still experimental and many mainstream languages have yet to adopt any standards that could seriously improve programming error message usability. This is perhaps because it is not clear from the literature as to what makes a programming error message "usable" [6]. We note that the usability and readability of error messages has been raised by several members of the computing education community during conference presentations, as part of recent Dagstuhl seminars [26, 40], and informally on computing education blogs. As an example of the 
latter, in a post on Becker's blog ${ }^{1}$ comparing approaches for measuring error message enhancement, Guzdial commented: "We need a robust measure and process for "enhancing" error messages. Simply making them longer is not an enhancement if students don't understand them. Any attempt to test "enhanced" error messages without first testing readability lacks validity."

Papers from the 1970s onward that provide any guidelines whatsoever for designing or evaluating programming error messages have almost unanimously listed 'readability' or some synonym as an essential guideline [1, 6, 23, 38, 41] without ever defining readability. In addition, these guidelines are generally vague, often conflicting, and rarely backed up with empirical evidence [6]. We claim that the readability of programming error messages is an essential characteristic of their potential usefulness and begin the process of gauging this readability.

In this paper we provide what we believe is the first analysis of the readability of programming error messages using human (expert, non-expert and student) raters. We establish evidence that a new metric designed for programming error messages is required. Our work is guided by the following research questions:

RQ1: Do experts, non-experts, and students assess the readability of programming error messages differently?

RQ2: Are error messages from different programming languages perceived to have different levels of readability?

\section{RELATED WORK}

Programming error messages (often called compiler error messages) [6] play a unique role in learning to program [45]. Namely, they complete an input/output (user/system) loop: the programmer inputs a program and (from the compiler/interpreter's perspective) messages are returned as feedback. These messages are typically a mixture of natural and programming language with additional technical terms. Exacerbating matters (at least in educational contexts), this feedback is provided regardless of whether an instructor is present and comes from a machine that many novices see as infallible [27].

\subsection{Difficulties with Programming Error Messages}

For over 50 years educators and researchers have been documenting the difficulties that programming error messages present, particularly to students. As early as 1965 systems were being built to have 'better' error messages [37], an area that has seen increased interest in recent years. In a recent large-scale survey of 107 papers on programming error messages, 10 were pre-2000, 31 were from 2000-2009, and the remaining 66 were from 2010-present, with 41 (35\% of all reviewed papers) published in the last 5 years [6]. Error messages are difficult to classify and analyze [9, 35]. They also show up in syllabi [7], in questions that practitioners have for researchers [15], and form an important part of the information that can be logged during programming sessions [34].

In 2016, McCall \& Kölling noted that Java error messages are not only confusing from the point of view of a novice, but probably for all programmers due two observations [29, p2]. First, a single error

\footnotetext{
${ }^{1}$ https://cszero.wordpress.com/2016/11/18/you-are-what-you-measure-enhancingcompiler-error-messages-effectively/
}

may, in a different context, produce different diagnostic messages; and second, the same diagnostic message may be produced by entirely different and distinct errors. Marceau, Fisler \& Krishnamurthi summed up the situation nicely [28, p3]: "In every environment, a mature programmer can usually point to at least a handful of favourite bad error responses. When they find out that the same environment is being used by novices, their laugh often hardens."

\subsection{Writing Error Messages Is Also Difficult}

Not only is interpreting programming error messages difficult, writing them is also. In 1976 Horning commented [23, p544]: "One of the hardest things to remember in designing error diagnostics is that you [the compiler-writer] do not know what the error was. Two (or more) pieces of information have been found to be inconsistent, but it cannot be said with certainty where the error lies." Although various guidelines for designing programming error messages have appeared in the literature, a recent study found that most did not directly address readability other than naming it (or a synonym) - most were concerned with context such as error location [6]. Additionally, most did not provide empirical evidence for their claims.

\subsection{Why Programming Error Messages Matter}

Barik et al. [3] present compelling justification for further research on error messages. They provide empirical evidence that students do read error messages (corroborated by $[19,33]$ ) and allocate a substantial portion of their total task to the reading and understanding of error messages. They also show that ironically, the difficulty of reading error messages is tantamount to that of source code itself.

This latter observation resonates with Buse \& Weimer who cited several sources noting that the act of reading code is the most timeconsuming of all maintenance activities [13]. This paints a picture contrary to common wisdom which often implies that programmers spend most of their time writing code-instead there is convincing evidence that programmers spend a lot of time reading code and reading error messages. Additionally, we hypothesize that everyone who has dealt with programming error messages has developed an intuitive sense that these messages matter, and are often less useful than they could be. We posit that readability has a large impact on message utility.

Further, as noted by Buse \& Weimer who cite at least a dozen papers on the topic, a consensus exists that code readability is an essential determining characteristic of code quality [13]. Aided by the findings of Barik et al., we view the importance of code readability to naturally extend to error messages. However there are likely differences between assessing the readability and usability of code and that of error messages.

Analysis of error messages and metrics that analyze them have been linked to traditional measures of academic performance [18, 42] and have provided important insights into novice programmer behavior [5, 24]. 'Improved' or 'enhanced' error messages have been incorporated into many tools such as novice code editors and automated assessment tools [3, 4, 8, 16, 19, 28, 32, 33, 41], often with positive effects such as reducing the number of errors overall [5]. Very recently it has been shown that changes in the presentation of programming error messages can affect student behavior [25], 
and so it is more than reasonable to assume that changes in the message content will also result in altered behaviors. For a recent detailed overview, see [6].

\subsection{Readability: Code \& Messages}

2.4.1 Source Code. Working memory plays an important role in source code comprehension as programmers need to work with several programming constructs (e.g., variables and methods) in parallel [22]. In studying code readability, Buse \& Weimer defined readability as "a human judgment of how easy a text is to understand" [13, p121]. They commented that the readability of a program is related to its maintainability, and is thus a critical factor in overall software quality [13]. They also provided a dozen references coming to a consensus that code readability is an essential characteristic of code quality. We claim that the readability of programming error messages is an essential characteristic of their potential usefulness. In exploring the applicability of traditional measures of prose readability to source code, Buse \& Weimer claimed that the readability of code is very different from that of natural languages. They also found that analyzing code snippets that were too short may obscure important readability considerations. This poses an immediate challenge for assessing the readability of error messages as they are relatively short by design. Other results on the length of code artifacts has revealed that shorter identifier names take longer to comprehend [22] again indicating that the relative shortness of error messages might be an issue in assessing their readability.

2.4.2 Programming Error Messages. Readability is clearly a very important part of the usability of programming error messages $[2,6]$. Readable messages are more memorable [20] and can increase the rate of error correction by utilizing the human brain's penchant for recognition over recall [41]. Previous research has argued that more readable error messages can decrease error rates and repeated errors [5], and reduce student frustration [36]. However, no previous studies have defined readability or provided a way to measure readability for programming error messages. Recent work has looked at error message structure. Barik et al. found that given a pair of error messages, developers significantly preferred messages that employ proper argument structure over deficient argument structure when neither offers a resolution, but will accept a deficient argument structure if it provides a resolution to the problem [1].

Existing metrics for the readability of natural-language prose appear inappropriate for assessing programming error messages [19]. The only guidelines for the readability of programming error messages that have been put forward in the literature are mostly vague and not easily measurable [6]. Most have to be inferred from more general usability guidelines, for instance "[Error Messages] should be expressed in plain language..." [31, p156].

\section{METHODOLOGY}

\subsection{Data Acquisition \& Analysis}

We found available datasets in three popular languages: Java (SE 7), C (gcc 5.4.0), and Python (3.6). For Java, we chose the 28,000 message dataset provided by [4], with the top 20 messages covering $89 \%$ of all messages. The Python dataset came from [44] and had
35,000 errors with the top 20 covering $99 \%$ of all messages. The C dataset came from [30] and contained 5,002 messages with the top 20 errors covering $95 \%$ of total. From each of these we extracted the 20 most common error messages. To give the reader an idea of how these messages look, several can be found in Tables 1 and 2 in Section 6.

\subsection{Human Analysis}

Given that existing English language readability metrics are not designed for assessing programming error messages, we set out to identify patterns which might help us devise our own readability metric. To this end, we conducted human surveys of 43 individuals. Five of these individuals (the "experts") hold university positions and terminal degrees in computer science and are based across North America, Europe and Australasia. Another five (the "non-experts") are non-computing professors/lecturers/instructors having very little or non-existent computing backgrounds, while still holding university positions in Europe and North America. The remaining 33 were first-year (week 4) students enrolled in a typical $\mathrm{CS} 1$ course being taught in $\mathrm{C}++$ at a North American university and thus had experienced programming error messages for a few weeks. At this university, while the course is taught with $\mathrm{C}++$, it might as well be $\mathrm{C}$ for their first semester because they are taught with a late-objects curriculum, which is why we chose to present the error messages in $\mathrm{C}$. The concepts the students had encountered thus far were: numeric types, basic mathematical and logical operators, conditionals, and nested conditionals.

The survey presented users with each of the twenty most frequent error messages from our three languages (Java, Python, and C), and asked each user to rate the readability of the message on a scale from 1 (least readable) to 10 (most readable). No detailed criteria were provided, allowing participants to make their own assessments as to what makes a message readable, and enabling us to see the extent to which ratings on such a scale are subjective across our three participant groups. We justify this as there is no clear definition of what readability means for programming error messages and as a first attempt in this area we wanted to see what unbiased responses would look like.

The "expert" group was also surveyed on their familiarity with the three languages, as well as asked (open-ended questions) about the criteria they applied when rating the error messages. This was done to gauge biases that might be present in the expert raters.

\section{RESULTS}

\subsection{Expert Analysis}

There was fairly low agreement between the readability scores of our five expert raters. Figure 1 shows the average of the readability scores assigned to the error messages from each language by each of the five experts (R1-R5). The y-axis indicates these, where a score of 10 represents "most readable". Krippendorff's alpha revealed a low inter-rater reliability (0.069). This is likely either due to bias among the expert raters, or because there isn't a consistent standard for readability among raters.

Anticipating bias in the experts, we also asked them to rate their C, Java, and Python knowledge when rating messages for readability. The three exact questions were "Rate your overall $<x>$ 


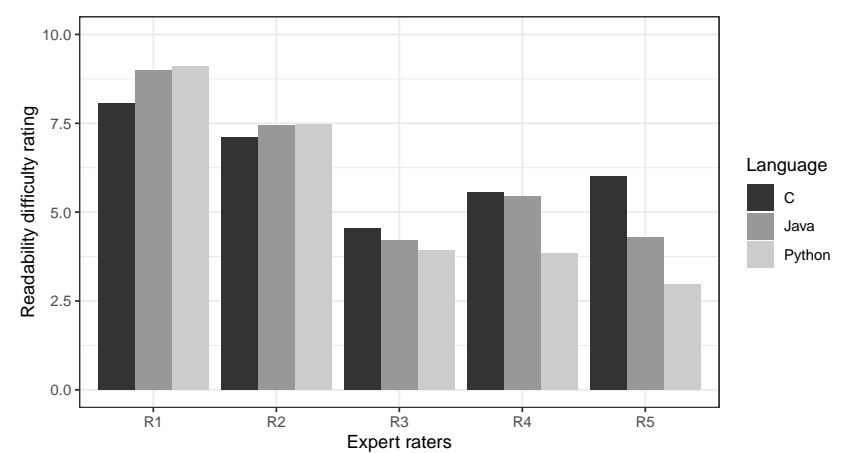

Figure 1: Readability of the most frequent error messages in $C$, Java and Python as rated by the five experts. The $y$-axis is an ordinal scale where 0 is least and 10 is most readable.

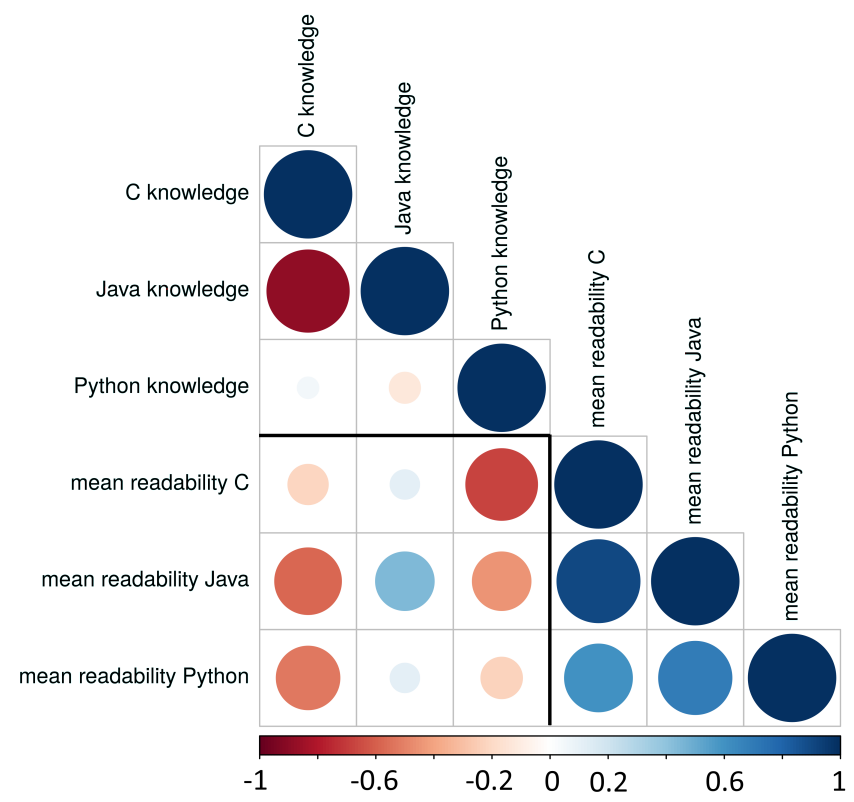

Figure 2: Correlation matrix showing correlation of language knowledge and message readability (by language) for experts (averaged). Scale is Spearman's correlation coefficient where $<-0.5 \&>0.5$ are considered relatively strong.

knowledge" where $<\mathrm{x}>$ was $\mathrm{C} / \mathrm{Python} / \mathrm{Java}$, and 1 was "none" and 5 was "expert". Figure 2 is a correlation matrix of average expert (self-rated) language knowledge and average expert-rated message readability (by language). It is clear that expert knowledge affects readability scores. The correlations in Figure 2 are most likely due to expert familiarity that comes with knowledge. Key observations are: 1) $\mathrm{C}$ knowledge is negatively correlated with Java and Python message readability; 2) Java knowledge is positively correlated with Java message readability; and 3) Python knowledge is strongly and moderately negatively correlated with $\mathrm{C}$ and Java message readability respectively. All three of these are plausible, however it is clear that more work is needed in this area.

\subsection{Non-Expert Peer Analysis}

We asked five professors in disciplines outside computing to do the same error message rating task as the experts. This was done to gauge the difference between those familiar with programming error messages and those who have no idea what one is. As firstyear students are likely between experts and non-experts, this also helped us position the student responses in relation to the experts. Additionally, although often tightly related to other qualities, readability does exist as a quality in and of itself. For instance, intricate poems or technical legal documents (in English) would be readable to non-experts to some extent. Therefore it may prove insightful to gauge the readability of error messages, as perceived by those who are not familiar with the context.

Figure 3 shows pairwise correlations between the experts (R1R5) and non-experts (C1-C5), across the 60 error message scores. The non-expert peer raters showed a high degree of agreement, but a low inter-rater reliability (Krippendorff's Alpha of 0.19). This is not a surprise because, although the non-expert peer raters agreed with each other more than the expert raters, they largely agreed the messages were unreadable, although not necessarily which ones.

\subsection{Comparing Expert \& Non-Expert Groups}

We found a highly statistically significant difference in the average readability of each error between the experts and non-experts (two $\mathrm{n}=60$ vectors): $\mathrm{t}(118)=4.634, \mathrm{p}<0.001$ (two tail $\mathrm{t}$-test). However none of the messages (individually) have significant differences between expert raters and non-expert raters.

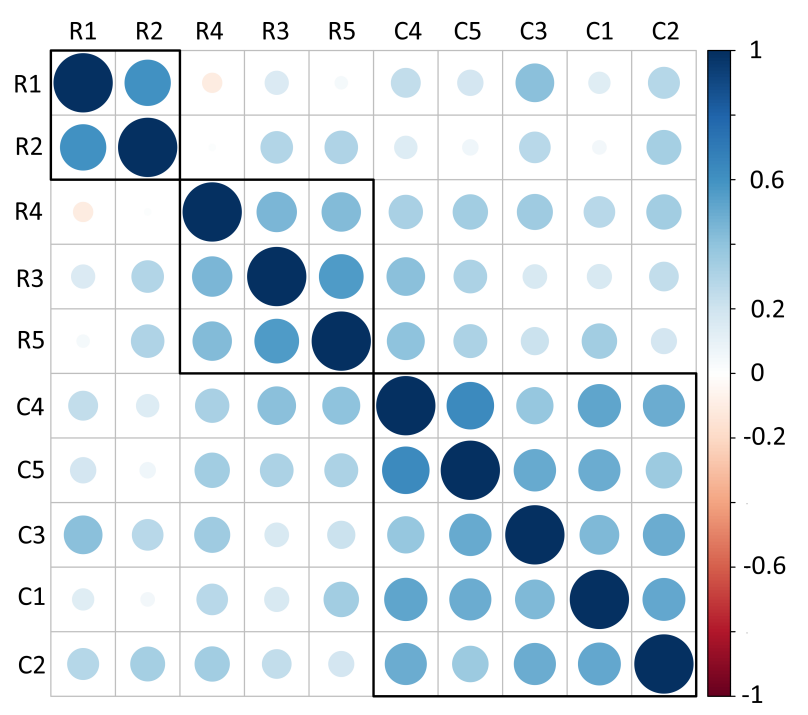

Figure 3: Correlation matrix showing experts (R1-R5) and non-experts (C1-C5) sorted using hierarchical clustering. Squares denote clusters. The experts form two clusters (within each cluster experts tend to agree) while the nonexperts form another cluster with relatively high agreement. Scale is Spearman's correlation coefficient where $<-0.5 \&>0.5$ are considered relatively strong. The only negative correlation is minor-in the expert group (between clusters). 
Figure 3 clearly shows two distinct groups, expert and non-expert. Within the expert group are two distinct subgroup clusters, R1-R2, and R3-R5. Agreement within these is relatively high showing that "readability" for different groups is distinct and any readability metric for programming error messages should take this into account.

\subsection{Student Analysis}

There were 33 student raters, two of whom rated all 60 messages at 10 and were excluded from analysis. We chose a higher number of students compared to experts and non-experts for two reasons: we are most interested in the student experience and, less importantly, convenience of sampling. We only use the expert and non-expert responses to situate the student responses. As discussed above, student raters were novice first-year students in the fourth week of CS1 (Introductory Programming). Perhaps unsurprisingly, the student raters had the highest variance and disagreement out of any rater group. The Krippendorff's alpha for inter-rater reliability among them was 0.008 , indicating a complete absence of agreement.

\subsection{Individual Error Messages}

Table 1 lists the 10 error messages with the highest average readability scores across all 41 raters (experts, peers and novices). Table 2 lists the 10 error messages with the lowest readability scores.

\section{FINDINGS}

RQ1: Do experts, non-experts and students assess the readability of programming error messages differently? Yes. These groups did assess the readability of error messages differently. This supports a hypothesis that different background knowledge and experience levels lead people to rate messages differently. Even within groups with similar backgrounds, such as computer science instructors, there are differences. Familiarity with a given programming language seems to have an impact on readability ratings. Interestingly, all raters tended to agree to a greater extent on what is more readable and to a lesser extent on what is less readable (see Figure 4). Given these results, a larger scale study would be helpful in establishing higher confidence in readability differences between messages and in elicitation of the factors that make messages readable. That information could be useful in discovering key factors that lead to more readable messages.

RQ2: Are error messages from different programming languages perceived to have different levels of readability? Yes. Messages were not perceived to be equally readable across languages. As a result, a future readability metric for programming error messages should take into account the readability of the language itself. Precisely how this is accomplished is a matter for future work, however there is some prior work on source code readability to build upon $[13,14]$.

\section{EXPLORATORY DISCUSSION}

There was little agreement between expert raters, yielding an exceptionally low inter-rater reliability value $(0.069$, on a scale from $0-1$ where 0.667 is considered the lowest conceivable limit for drawing even tentative conclusions [21]). Pairwise correlations show that experts fell into two clusters, with moderate agreement within clusters, but negative correlation between them-a somewhat surprising result. However, previous work shows that expert judgments in this domain are not always reliable [39]. In 2014 Brown \& Altadmri analyzed over 100,000 student programming errors and surveyed 76 experts, finding that educators formed a weak consensus about which mistakes are most frequent, their rankings bore only moderate relationship to the student data, and that educators' experience had no effect on the level of agreement [10]. Their results raise questions about expert ratings on error messages (by extension through the errors themselves) and mirror our findings that expert ratings are insufficient for determining the readability of error messages.

Despite representing three different languages, the individual error messages in Table 1 generally appear to be more alike than they do compared to those in Table 2 and vice-versa. Examination suggests three factors which may play a role in perceived readability: message length, tone, and the density of jargon/acronyms.

First, shorter messages tend to be rated as more readable. The median number of characters per message in Table 1 (24) is almost half that of Table 2 (45). However, there are clear exceptions to this which are puzzling. An example involves the message with rank 2 (high readability) - '(' expected) - and the message with rank 59 (poor readability) - expected ' $=$ '. On the surface these messages are almost identical, consisting of the same word, but each with a different symbol appearing in a different order. Pedagogically it is interesting that one of the most frequent messages novices encounter - ';' expected - is ranked as the most readable.

Second, message tone appears to be a factor. Many of the messages in Table 2 use negative phrasing: "bad", "invalid", "cannot be", "incompatible", "unexpected", etc., yet this kind of language is almost entirely absent from the messages in Table 1 . This finding provides some initial empirical support for earlier suggested guidelines on both system and compiler messages which recommend avoiding words with a negative valence [6, 38, 41].

Third, it also appears that messages with more jargon, technical terms, and acronyms are perceived to be less readable. As an example, the message ranked 60 contains quite a bit of jargon. It was unanimously given the lowest rating by all five non-experts, and the only message across 180 data points (three groups of raters; 60 messages, averaged, per group) with a standard deviation of 0 .

Finally, although agreement between all raters was low when calculated across all messages, the level of agreement is positively correlated with the readability of the message as illustrated in Figure 4. In general, there was more agreement (lower SD) for messages ranked as more readable. This is evidence that identifying messages that are easy to read seems to be a simpler, less subjective process than identifying messages which are not.

\section{LIMITATIONS}

This work has known limitations, many of which we felt must be accepted at such an early stage of such subjective work. These observations should help form criteria for future work.

The process used to rate readability was knowingly subjective and may account for the generally low agreement observed. As noted in Section 3.2, one of the difficulties at this early stage of exploration is that there is no clear definition of what readability 


\begin{tabular}{|c|c|c|c|c|c|c|}
\hline \multirow[b]{2}{*}{ Rank } & \multirow[b]{2}{*}{ Language } & \multirow[b]{2}{*}{ Programming Error Message } & \multicolumn{4}{|c|}{ Mean (Standard Deviation) } \\
\hline & & & Experts & Non-experts & Students & Overall \\
\hline 1 & Java & ‘; expected & $6.40(1.52)$ & $7.40(2.41)$ & $9.17(1.79)$ & $8.59(2.07)$ \\
\hline 2 & Java & '(' expected & $6.40(2.07)$ & $7.00(2.83)$ & $9.17(1.47)$ & $8.54(2.02)$ \\
\hline 3 & Java & 'else' without 'if' & $7.40(1.82)$ & $5.40(3.05)$ & $9.24(1.83)$ & $8.51(2.37)$ \\
\hline 4 & Java & ']' expected & $6.20(2.39)$ & $7.20(3.03)$ & $9.10(1.52)$ & $8.49(2.11)$ \\
\hline 5 & $\mathrm{C}$ & 'else' without a previous 'if' & $8.60(1.14)$ & $4.40(3.78)$ & $9.00(2.14)$ & $8.36(2.72)$ \\
\hline 6 & Java & ')' expected & $6.00(2.12)$ & $7.40(2.41)$ & $8.90(1.92)$ & $8.33(2.20)$ \\
\hline 7 & $\mathrm{C}$ & no such file or directory & $8.40(1.52)$ & $8.40(2.61)$ & $8.07(2.49)$ & $8.15(2.36)$ \\
\hline 8 & $\mathrm{C}$ & expected $<\mathrm{x}>$ before $<\mathrm{y}>$ & $7.00(1.41)$ & $5.80(3.35)$ & $8.34(1.74)$ & $7.85(2.11)$ \\
\hline 9 & Python & Missing parentheses in call to 'print' & $7.60(1.82)$ & $6.60(2.88)$ & $8.10(2.47)$ & $7.85(2.44)$ \\
\hline 10 & Python & expected an indented block & $7.40(2.51)$ & $6.60(3.85)$ & $8.03(2.04)$ & $7.77(2.36)$ \\
\hline
\end{tabular}

Table 1: Ten error messages with the highest average readability across all raters (experts, non-experts and students).

\begin{tabular}{|c|c|c|c|c|c|c|}
\hline \multirow[b]{2}{*}{ Rank } & \multirow[b]{2}{*}{ Language } & \multirow[b]{2}{*}{ Programming Error Message } & \multicolumn{4}{|c|}{ Mean (Standard Deviation) } \\
\hline & & & Experts & Non-experts & Students & Overall \\
\hline 51 & Java & bad operand types for binary operator ' $*$ operator*' & $6.40(1.67)$ & $1.40(0.55)$ & $5.93(2.40)$ & $5.41(2.65)$ \\
\hline 52 & $\mathrm{C}$ & invalid type argument of $\langle\ldots\rangle$ & $4.80(2.68)$ & $4.60(2.70)$ & $5.66(2.42)$ & $5.41(2.46)$ \\
\hline 53 & Python & positional argument follows keyword argument & $5.20(3.27)$ & $2.80,(1.64)$ & $5.55(2.50)$ & $5.15(2.62)$ \\
\hline 54 & Java & $\begin{array}{l}\text { incompatible types OR incompatible types: } \\
* \text { type* cannot be converted to *type* }\end{array}$ & $4.80(3.11)$ & $1.60(0.89)$ & $5.69(2.85)$ & $5.05(3.00)$ \\
\hline 55 & Python & unexpected EOF while parsing & $5.00(2.74)$ & $1.80(0.84)$ & $5.52(3.16)$ & $4.97(3.12)$ \\
\hline 56 & Python & EOL while scanning string literal & $4.00(3.00)$ & $1.20(0.45)$ & $5.28(2.99)$ & $4.59(3.08)$ \\
\hline 57 & Python & EOF while scanning triple-quoted string literal & $3.80(3.27)$ & $1.40(0.89)$ & $5.07(2.85)$ & $4.44(2.96)$ \\
\hline 58 & $\mathrm{C}$ & invalid operands to binary $<\mathrm{x}>$ (have $<\mathrm{y}>$ and $<\mathrm{z}>$ ) & $4.20,(2.28)$ & $1.40,(0.89)$ & $4.83,(2.52)$ & $4.31,(2.58)$ \\
\hline 59 & $\mathrm{C}$ & expected ' $=$ ’ & $2.60(2.51)$ & $3.00(2.92)$ & $4.03(2.63)$ & $3.72(2.64)$ \\
\hline 60 & Python & (unicode error 'unicodeescape' codec can't decode bytes & $2.20(1.30)$ & $1.00(0.00)$ & $3.93(2.62)$ & $3.33(2.53)$ \\
\hline
\end{tabular}

Table 2: Ten error messages with the lowest average readability across all raters (experts, non-experts and students).

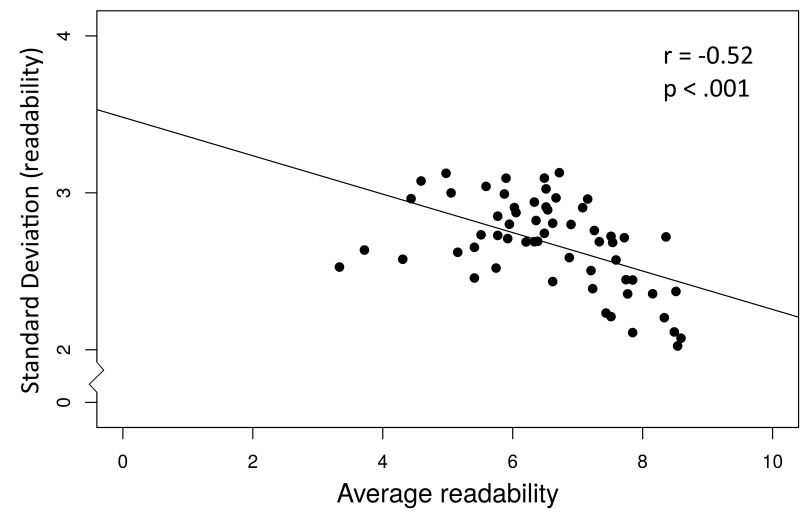

Figure 4: Standard deviation of readability vs average readability (0 low - 10 high). Data points are the means of each programming error message across all raters (expert, nonexpert and student). The relatively high correlation indicates that all raters agree more strongly for more readable error messages, and disagree more for less readable messages.

means for programming error messages. Therefore, raters likely brought different assumptions with them. Since error messages are generated in response to code errors, judging the readability of a message is arguably influenced by the context of the code itself. Although this code was not shown to raters, it is possible that some unconsciously took this into account more than others. Ratings may have also been influenced by explicit knowledge of certain messages if the raters were familiar with them.

This subjectivity may have particularly impacted our expert raters. There was likely a familiarity bias for which an individual tended to code messages in the language with which they were most familiar differently from the other languages. Certainly the correlations we observed in Figure 2, between language familiarity and error message readability, show that the relationship between knowledge of a language and the perceived readability of its messages is complex. Our results highlight the need for identifying factors that can contribute to a more reliable and objective metric.

In addition, the low agreement in ratings overall may have been exacerbated by the small number of raters involved in our study. Our expert raters clustered into two small groups, each having very similar backgrounds and familiarity with distinct languages. A larger pool of raters, particularly experts with more heterogeneous backgrounds, would help to mitigate this bias.

Finally, it should be noted that programming error messages are almost always tied directly to a context in code. They are the the result of trying to compile or execute code which violates the 
language specification. They should describe that violation including the location and other helpful information. This might make evaluating the readability of the message text in abstract difficult. However, we propose that the messages themselves still need to be readable, regardless of context. Furthermore, compiler writers and architects write error messages in abstract with the understanding that many actual errors could cause the same message to appear. Therefore, we believe that it is misguided to suggest the messages are so tied to context that seeking to understand their readability in a limited context, or by non-experts, is fruitless.

\section{CONCLUSION}

Programmers, particularly novices, can spend significant time trying to decipher error messages. Researchers suggesting ways to make such messages more effective often advocate that error messages should be more "readable." However, no research has presented a working definition of readability for programming error messages or how it can be measured. In this paper, we provide two important contributions: (1) human (expert, non-expert, and novice student) ratings of the readability of error messages from three popular languages; and (2) insights derived from our observations to guide future efforts for creating a readability metric for programming error messages.

We identified several factors that may impact how readability is perceived. These include message length, tone, and the use of jargon and acronyms. While these factors seem plausible and are related to guidelines identified in prior literature, they are derived from our analysis of only a small subset of the messages in our study-the ten most and ten least readable overall (Table $1 \&$ Table 2). Future work should consider a much larger set of messages and determine the extent to which these factors do impact readability.

We close by noting that more research on this subject is required. As we have argued, establishing a valid metric for error message readability is fundamental to future progress in creating more usable error messages for all programmers, but especially novices.

\section{REFERENCES}

[1] Titus Barik. 2018. Error Messages as Rational Reconstructions. Ph.D. Dissertation. North Carolina State University, Raleigh. https://repository.lib.ncsu.edu/handle/ $1840.20 / 35439$

[2] Titus Barik, Denae Ford, Emerson Murphy-Hill, and Chris Parnin. 2018. How Should Compilers Explain Problems to Developers?. In Proceedings of the 2018 26th ACM foint Meeting on European Software Engineering Conference and Symposium on the Foundations of Software Engineering (Lake Buena Vista, FL, USA) (ESEC/FSE 2018). ACM, New York, NY, USA, 633-643. https://doi.org/10.1145/3236024. 3236040

[3] Titus Barik, Justin Smith, Kevin Lubick, Elisabeth Holmes, Jing Feng, Emerson Murphy-Hill, and Chris Parnin. 2017. Do Developers Read Compiler Error Messages?. In Proceedings of the 39th International Conference on Software Engineering (Buenos Aires, Argentina) (ICSE '17). IEEE Press, Piscataway, NJ, USA, 575-585. https://doi.org/10.1109/ICSE.2017.59

[4] Brett A Becker. 2015. An Exploration Of The Effects Of Enhanced Compiler Error Messages For Computer Programming Novices. Masters Thesis. Dublin Institute of Technology. https://doi.org/10.13140/RG.2.2.26637.13288

[5] Brett A. Becker. 2016. An Effective Approach to Enhancing Compiler Error Messages. In Proceedings of the 47th ACM Technical Symposium on Computing Science Education (Memphis, Tennessee, USA) (SIGCSE '16). ACM, New York, NY, USA, 126-131. https://doi.org/10.1145/2839509.2844584

[6] Brett A. Becker, Paul Denny, Raymond Pettit, Durell Bouchard, Dennis J. Bouvier, Brian Harrington, Amir Kamil, Amey Karkare, Chris McDonald, Peter-Michael Osera, Janice L. Pearce, and James Prather. 2019. Compiler Error Messages Considered Unhelpful: The Landscape of Text-Based Programming Error Message Research. In Proceedings of the Working Group Reports on Innovation and
Technology in Computer Science Education (Aberdeen, Scotland UK) (ITiCSEWGR '19). Association for Computing Machinery, New York, NY, USA, 177-210. https://doi.org/10.1145/3344429.3372508

[7] Brett A. Becker and Thomas Fitzpatrick. 2019. What Do CS1 Syllabi Reveal About Our Expectations of Introductory Programming Students?. In Proceedings of the 50th ACM Technical Symposium on Computer Science Education (Minneapolis, MN, USA) (SIGCSE '19). Association for Computing Machinery, New York, NY, USA, 1011-1017. https://doi.org/10.1145/3287324.3287485

[8] Brett A. Becker, Graham Glanville, Ricardo Iwashima, Claire McDonnell, Kyle Goslin, and Catherine Mooney. 2016. Effective Compiler Error Message Enhancement for Novice Programming Students. Computer Science Education 26, 2-3 (2016), 148-175. https://doi.org/10.1080/08993408.2016.1225464

[9] Brett A Becker and Catherine Mooney. 2016. Categorizing Compiler Error Messages With Principal Component Analysis. In 12th China-Europe International Symposium on Software Engineering Education (CEISEE 2016), Shenyang, China, 28-29 May 2016.

[10] Neil C.C. Brown and Amjad Altadmri. 2014. Investigating Novice Programming Mistakes: Educator Beliefs vs. Student Data. In Proceedings of the Tenth Annual Conference on International Computing Education Research (Glasgow, Scotland, United Kingdom) (ICER '14). ACM, New York, NY, USA, 43-50. https://doi.org/ $10.1145 / 2632320.2632343$

[11] P. J. Brown. 1982. My System Gives Excellent Error Messages - Or Does It? Software: Practice and Experience 12, 1 (Jan 1982), 91-94. https://doi.org/10.1002/ spe. 4380120110

[12] P. J. Brown. 1983. Error Messages: The Neglected Area of the Man/Machine Interface. Commun. ACM 26, 4 (Apr 1983), 246-249. https://doi.org/10.1145/ 2163.358083

[13] Raymond P.L. Buse and Westley R. Weimer. 2008. A Metric for Software Readability. In Proceedings of the 2008 International Symposium on Software Testing and Analysis (Seattle, WA, USA) (ISSTA '08). ACM, New York, NY, USA, 121-130. https://doi.org/10.1145/1390630.1390647

[14] Ron Coleman. 2018. Aesthetics Versus Readability of Source Code. International Journal of Advanced Computer Science and Applications 9, 9 (2018), 12-18.

[15] Paul Denny, Brett A. Becker, Michelle Craig, Greg Wilson, and Piotr Banaszkiewicz. 2019. Research This! Questions That Computing Educators Most Want Computing Education Researchers to Answer. In Proceedings of the 2019 ACM Conference on International Computing Education Research (Toronto ON, Canada) (ICER '19). Association for Computing Machinery, New York, NY, USA, 259-267. https://doi.org/10.1145/3291279.3339402

[16] Paul Denny, Andrew Luxton-Reilly, and Dave Carpenter. 2014. Enhancing Syntax Error Messages Appears Ineffectual. In Proceedings of the 19th Conference on Innovation and Technology in Computer Science Education (Uppsala, Sweden) (ITiCSE '14). ACM, New York, NY, USA, 273-278. https://doi.org/10.1145/2591708. 2591748

[17] Paul Denny, Andrew Luxton-Reilly, and Ewan Tempero. 2012. All Syntax Errors Are Not Equal. In Proceedings of the 17th ACM Annual Conference on Innovation and Technology in Computer Science Education (Haifa, Israel) (ITiCSE '12). ACM, New York, NY, USA, 75-80. https://doi.org/10.1145/2325296.2325318

[18] Paul Denny, Andrew Luxton-Reilly, Ewan Tempero, and Jacob Hendrickx. 2011. Understanding the Syntax Barrier for Novices. In Proceedings of the 16th Annual foint Conference on Innovation and Technology in Computer Science Education (Darmstadt, Germany) (ITiCSE '11). ACM, New York, NY, USA, 208-212. https: //doi.org/10.1145/1999747.1999807

[19] Paul Denny, James Prather, and Brett A. Becker. 2020. Error Message Readability and Novice Debugging Performance. In Proceedings of the 2020 ACM Conference on Innovation and Technology in Computer Science Education (Trondheim, Norway) (ITiCSE '20). Association for Computing Machinery, New York, NY, USA, 480-486. https://doi.org/10.1145/3341525.3387384

[20] T. Flowers, C.A. Carver, and J. Jackson. 2004. Empowering Students and Building Confidence in Novice Programmers Through Gauntlet. In 34th ASEE/IEEE Annual Frontiers in Education (FIE '04), Vol. 1. IEEE, Savannah, GA, USA, T3H/10-T3H/13. https://doi.org/10.1109/fie.2004.1408551

[21] Cristina M. Giannantonio. 2010. Book Review: Krippendorff, K. (2004). Content Analysis: An Introduction to Its Methodology (2nd ed.). Thousand Oaks, CA: Sage. Organizational Research Methods 13, 2 (2010), 392-394. https://doi.org/10. 1177/1094428108324513 arXiv:https://doi.org/10.1177/1094428108324513

[22] Johannes C Hofmeister, Janet Siegmund, and Daniel V Holt. 2019. Shorter Identifier Names Take Longer to Comprehend. Empirical Software Engineering 24, 1 (2019), 417-443.

[23] James J Horning. 1976. What the Compiler Should Tell the User. In Compiler Construction: An Advanced Course, G Goos and J Hartmanis (Eds.). SpringerVerlag, Berlin-Heidelberg, 525-548.

[24] Matthew C. Jadud. 2006. An Exploration of Novice Compilation Behaviour in Bluef. Ph.D. Dissertation. University of Kent at Canterbury. https://jadud.com/dl/pdf/ jadud-dissertation.pdf

[25] Ioannis Karvelas, Annie Li, and Brett A. Becker. 2020. The Effects of Compilation Mechanisms and Error Message Presentation on Novice Programmer Behavior. In Proceedings of the 51st ACM Technical Symposium on Computer Science Education 
(Portland, OR, USA) (SIGCSE '20). Association for Computing Machinery, New York, NY, USA, 759-765. https://doi.org/10.1145/3328778.3366882

[26] Amy J Ko, Shriram Krishnamurthi, Gail C Murphy, and Janet Siegmund. 2016 Human-Centric Development of Software Tools (Dagstuhl Seminar 15222). In Dagstuhl Reports, Vol. 5. Schloss Dagstuhl-Leibniz-Zentrum fuer Informatik.

[27] Michael J. Lee and Amy J. Ko. 2011. Personifying Programming Tool Feedback Improves Novice Programmers' Learning. In Proceedings of the Seventh International Workshop on Computing Education Research (Providence, Rhode Island, USA) (ICER '11). ACM, New York, NY, USA, 109-116. https://doi.org/10.1145/ 2016911.2016934

[28] Guillaume Marceau, Kathi Fisler, and Shriram Krishnamurthi. 2011. Mind Your Language: On Novices' Interactions with Error Messages. In 10th SIGPLAN Symposium on New Ideas, New Paradigms, and Reflections on Programming and Software - ONWARD '11. ACM, Portland, Oregon, USA, 3-17. https //doi.org/10.1145/2048237.2048241

[29] David Mccall. 2016. Novice Programmer Errors-Analysis and Diagnostics. Ph.D. Dissertation. The University of Kent. https://kar.kent.ac.uk/id/eprint/61340

[30] Cormac Murray. 2019. An Analysis of Programming Processs Data in an Introductory Programming Module: Factors Influencing Success. Master's thesis. University College Dublin, Dublin, Ireland.

[31] Jakob Nielsen. 1994. Enhancing the explanatory power of usability heuristics. In Conference companion on Human factors in computing systems - CHI '94. ACM Press, New York, New York, USA, 152-158. https://doi.org/10.1145/259963.260333

[32] Raymond S. Pettit, John Homer, and Roger Gee. 2017. Do Enhanced Compiler Error Messages Help Students? Results Inconclusive. In Proceedings of the 48th ACM Technical Symposium on Computer Science Education. Seattle, USA, 465-470. https://doi.org/10.1145/3017680.3017768

[33] James Prather, Raymond Pettit, Kayla Holcomb McMurry, Alani Peters, John Homer, Nevan Simone, and Maxine Cohen. 2017. On Novices' Interaction with Compiler Error Messages. In Proceedings of the 2017 ACM Conference on International Computing Education Research. 74-82. https://doi.org/10.1145/3105726. 3106169

[34] Thomas W. Price, David Hovemeyer, Kelly Rivers, Ge Gao, Austin Cory Bart, Ayaan M. Kazerouni, Brett A. Becker, Andrew Petersen, Luke Gusukuma, Stephen H. Edwards, and David Babcock. 2020. ProgSnap2: A Flexible Format for Programming Process Data. In Proceedings of the 2020 ACM Conference on Innovation and Technology in Computer Science Education (Trondheim, Norway) (ITiCSE '20). Association for Computing Machinery, New York, NY, USA, 356-362. https://doi.org/10.1145/3341525.3387373
[35] David Pritchard. 2015. Frequency Distribution of Error Messages. In Proceedings of the 6th Workshop on Evaluation and Usability of Programming Languages and Tools. 1-8. https://doi.org/10.1145/nnnnnnn.nnnnnnn arXiv:1509.07238v1

[36] Peter C. Rigby and Suzanne Thompson. 2005. Study of Novice Programmers using Eclipse and Gild. In Proceedings of the 2005 OOPSLA Workshop on Eclipse Technology eXchange. ACM, San Diego, California, 105-109. https://doi.org/10. 1145/1117696.1117718

[37] Saul Rosen, Robert A. Spurgeon, and Joel K. Donnelly. 1965. PUFFT - The Purdue University Fast FORTRAN Translator. Commun. ACM 8, 11 (nov 1965), 661-666. https://doi.org/10.1145/365660.365671

[38] Ben Shneiderman. 1982. Designing Computer System Messages. Commun. ACM 25, 9 (1982), 610-611. https://doi.org/10.1145/358628.358639

[39] James C. Spohrer and Elliot Soloway. 1986. Novice Mistakes: Are the Folk Wisdoms Correct? Commun. ACM 29, 7 (July 1986), 624-632. https://doi.org/10. $1145 / 6138.6145$

[40] Andreas Stefik, Bonita Sharif, Brad. A. Myers, and Stefan Hanenberg. 2018. Evidence About Programmers for Programming Language Design (Dagstuhl Seminar 18061). Dagstuhl Reports 8, 2 (2018), 1-25. https://doi.org/10.4230/DagRep.8.2.1

[41] V. Javier Traver. 2010. On Compiler Error Messages: What They Say and What They Mean. Advances in Human-Computer Interaction 2010 (2010), 1-26. https: //doi.org/10.1155/2010/602570

[42] Christopher Watson, Frederick W.B. Li, and Jamie L. Godwin. 2014. No Tests Required: Comparing Traditional and Dynamic Predictors of Programming Success. In Proceedings of the 45th ACM Technical Symposium on Computer Science Education (Atlanta, Georgia, USA) (SIGCSE '14). ACM, New York, NY, USA, 469-474. https://doi.org/10.1145/2538862.2538930

[43] Richard L. Wexelblat. 1976. Maxims for Malfeasant Designers, or How to Design Languages to Make Programming As Difficult As Possible. In Proceedings of the 2nd International Conference on Software Engineering (San Francisco, California, USA) (ICSE '76). IEEE Computer Society Press, Los Alamitos, CA, USA, 331-336. http://dl.acm.org/citation.cfm?id=800253.807695

[44] Alexander William Wong, Amir Salimi, Shaiful Chowdhury, and Abram Hindle. 2019. Syntax and Stack Overflow: A Methodology for Extracting a Corpus of Syntax Errors and Fixes. (jul 2019). arXiv:1907.07803 http://arxiv.org/abs/1907. 07803

[45] John Wrenn and Shriram Krishnamurthi. 2017. Error Messages are Classifiers: A Process to Design and Evaluate Error Messages. In Proceedings of the 2017 ACM SIGPLAN International Symposium on New Ideas, New Paradigms, and Reflections on Programming and Software. ACM New York, NY, USA, Vancouver, BC, Canada, 134-147. https://doi.org/10.1145/3133850.3133862 